\title{
Atomistic Modeling of Mechanical Characteristics of CNT-Polyethylene with Interfacial Covalent Interaction
}

\author{
Qi-lin Xiong' and Xiao-geng Tian ${ }^{2}$ \\ ${ }^{1}$ School of Civil Engineering \& Mechanics, Huazhong University of Science \& Technology, 1037 Luoyu Road, Wuhan 430074, China \\ ${ }^{2}$ State Key Laboratory for Mechanical Structure Strength and Vibration, Xian Jiaotong University, Xian 710049, China \\ Correspondence should be addressed to Qi-lin Xiong; xiongql@hust.edu.cn
}

Received 30 January 2015; Accepted 20 March 2015

Academic Editor: Ottorino Ori

Copyright (c) 2015 Q.-1. Xiong and X.-g. Tian. This is an open access article distributed under the Creative Commons Attribution License, which permits unrestricted use, distribution, and reproduction in any medium, provided the original work is properly cited.

\begin{abstract}
The mechanical properties of carbon nanotube- (CNT-) reinforced polyethylenes (PE) with interfacial covalent bonded interaction are investigated using molecular dynamics simulations. A reactive force field for hydrocarbons (ReaxFF) is used in the nanocomposite system. Through a series of the tensile and pullout tests of carbon nanotube-reinforced polyethylene, Young's modulus and the interfacial shear stress of the nano-reinforced polyethylene are obtained. The comparisons between the MD results of this work and the relevant experimental data of the existing literature are made and the results show that the interfacial covalent bonded interaction between CNTs and the polymer matrix is indispensable. The bond interaction plays the main role in the load transfer of nanocomposites. In addition, the influences of carbon nanotube embedded length and diameter on the interfacial mechanical properties also are studied.
\end{abstract}

\section{Introduction}

Carbon nanotubes- (CNTs-) reinforced various nanocomposites are attracting more and more attention due to their superior mechanical, thermal, and electrical properties, making them distinguishable from bulk materials [1-5]. It is well known that load transfer efficiency from the polymer matrix to the reinforcement plays an important role in the mechanical properties of the reinforced nanocomposite. To enhance the mechanical properties of the reinforced nanocomposite, the improvement of load transfer efficiency is indispensable. And the main issue of effectively improving the interfacial load transfer of nanocomposites is the interfacial bonding between the individual CNTs and the polymeric matrix. The significant number of methods in the literature to improve the interfacial load transfer of nanocomposites mainly is categorized into the following two types: noncovalent and covalent. But the covalent improvement methods always give the nano-reinforced composites the better results [6].

The pullout experiments of individual carbon nanotubes from polymer matrices have been done to evaluate the interfacial shear strength of the nano-reinforced composite system by Cooper et al. [7]. Koval'chuk et al. [8] studied the effect of carbon nanotube functionalization on the structural and mechanical properties of polypropylene/MWCNT composites and found that a better dispersion of the nanotube reinforcements within the polymer matrix can be achieved with covalent interaction. Frankland et al. [9] investigated the influence of chemical cross-links on the shear strength of carbon nanotube-polymer interfaces by using molecular simulations; the conclusion showed that the chemical functionalization of the nanotube surface can improve and enhance the interfacial adhesion. Lachman and Wagner [10] studied the effect of the molecular nature of the interface between an epoxy matrix and multiwalled carbon nanotubes (CNTs) on the mechanical properties of the resultant nanocomposites and found that the nanocomposite toughness is found to increase with enhanced interfacial adhesion, which is opposite to what is usually observed in traditional fiber-based composites. Buffa et al. [11] studied the effect of nanotube functionalization on the properties of single-walled carbon nanotube/polyurethane composites by experiments, and 
the results show that the functionalization of the tubes results in increases in Young's modulus, tensile strength, and strain at failure and also destroys the inherent electrical conductivity of the tubes as well. Zheng et al. [12, 13] investigated the influence of sidewall modification on the interfacial bonding between the SWNTs and polymer and the influence of chemical functionalization on the interfacial bonding characteristics of single-walled nanotubes- (SWNTs-) reinforced polymer composites using molecular mechanics (MM) and molecular dynamics (MD) simulations. The results show that appropriate functionalization of nanotubes at low densities of functionalized carbon atoms drastically increases their interfacial bonding and shear stress between the nanotubes and the polymer matrix. But the association of single-walled nanotube (SWNT) with polyethylene (PE) molecule is still noncovalent.

Barber et al. [14] performed reproducible nano-pullout experiments of separating a carbon nanotube from a solid polymer matrix to measure the force by using atomic force microscopy and found the separation force is remarkably high and the experimental interfacial strength is much higher than the interfacial strength of a CNT-polyethylene system predicted using van der Waals interactions by computer modeling. The results infer that covalent bonding exists between the polymer matrix and CNT; that is, the bonding model should consider chemical as well as physical interactions. Recently, the various implement approaches of improving the covalent bonding between CNTs with polymers through chemical modification are reported [15-20]. Recently, Xiong and Tian investigated the interfacial mechanical properties of carbon nanotube-(CNT-) reinforced silicon nanocomposites by using molecular dynamics simulation method [21]. But the computationally modeling study of the covalent bonding between CNTs with polymers has been rarely reported.

In the present work, we apply molecular dynamics simulation and account for the covalent bonded interaction, van der Waals interaction, and the electrostatic interaction in the interface of nanocomposite by employing the reactive force field (ReaxFF) to investigate the effect of the interfacial covalent bond on the mechanical properties of carbon nanotube-reinforced polyethylene. Through a series of the tensile and pullout tests of nano-reinforced composite, Young's modulus and the interfacial shear stress of the nanoreinforced polyethylene are obtained.

\section{Process of Simulation}

2.1. Force Field (ReaxFF [22]). ReaxFF uses a general relationship between bond distance and bond order on one hand and between bond order and bond energy on the other hand that leads to proper dissociation of bonds to separated atoms. Other valence terms present in the force field (angle and torsion) are defined in terms of the same bond orders so that all these terms go to zero smoothly as bonds break. In addition, ReaxFF has Coulomb and Morse (van der Waals) potentials to describe nonbond interactions between all atoms (no exclusions). The parameters were derived from quantum chemical calculations on bond dissociation and reactions of small molecules plus heat of formation and geometry data for a number of stable hydrocarbon compounds. ReaxFF provides a good description of these data.

In general, the reactive force field divides the system total energy up into various partial energy contributions, as shown in the following [22]:

$$
\begin{aligned}
E_{\text {system }}= & E_{\text {bond }}+E_{\text {over }}+E_{\text {under }}+E_{\mathrm{val}}+E_{\text {pen }} \\
& +E_{\text {tors }}+E_{\text {conj }}+E_{\text {vdWaals }}+E_{\text {Coulomb }}
\end{aligned}
$$

where $E_{\text {bond }}$ is the bond energy of nanosystem, $E_{\text {over }}$ and $E_{\text {under }}$ are the bond energy penalty of the atom over coordination and under coordination, $E_{\mathrm{val}}$ denotes the valence angle energy of nanosystem, $E_{\text {pen }}$ is the angle penalty energy of the atom over coordination and under coordination, $E_{\text {tors }}$ is the torsion angles energy of nanosystem, $E_{\text {conj }}$ is the contribution of conjugation effects to the molecular energy, $E_{\mathrm{vdWaals}}$ denotes nonbonded van der Waals interactions energy of nanosystem, and $E_{\text {Coulomb }}$ is the coulomb interactions energy of nanosystem.

The detailed forms of expression for every energy contribution and all the parameters in the above formulas can be obtained from [22]. The detailed values for the hydrocarbon parameters of the MD simulations in the present work are taken from [22].

To validate ReaxFF force field used in the present study, the two experiments of the tensile test of a singlewalled carbon nanotube from [23] and a multiwalled carbon nanotube from [24] were simulated. Figures 1(a) and 1(b) compare the MD predicted stress-strain curve profiles of a two-walled carbon nanotube and a single-walled carbon nanotube with those measured data from the published documents. Except for the difference between the MD simulation and the experimental data at large strain for multiwalled carbon nanotubes, the agreement between MD predictions and experimental measurements is good, demonstrating the effectiveness and accuracy of ReaxFF force field. And the discrepancies between the MD simulation and the experimental data can be attributed to differences in the different multiwalled CNTs investigated and the presence of some defects in the experimental samples.

2.2. Molecular Model and Molecular Dynamics Simulations. A schematic diagram of the steps involved in the MD modelling of the pullout model is illustrated in Figure 2. We have generated the single polymer molecular chain and CNT by using VMD [26]. Subsequently, Packmol software [27] is utilized to create a targeted molecular model for MD simulations. Large-Scale Atomic Molecular Massively Parallel Simulator (LAMMPS) [28] is used to perform all MD simulations and postprocessing of the simulations was performed using VMD.

Each polyethylene chain consists of 20 repeating units of $-\mathrm{CH}_{2}$; finally adding hydrogen atoms at both two ends of each polyethylene chain makes the polymer neutral. The length and diameter of this fragment SWNTs $(6,6)$ are $4.92 \mathrm{~nm}$ and $0.815 \mathrm{~nm}$, which are selected for the simulations of SWNT/PE composites. SWNTs $(6,6)$ are placed in the center of model box of $2.7 \mathrm{~nm} \times 2.7 \mathrm{~nm} \times 5.0 \mathrm{~nm}$ and then $36 \mathrm{PE}$ chains are paced into the model box randomly to obtain the initial 


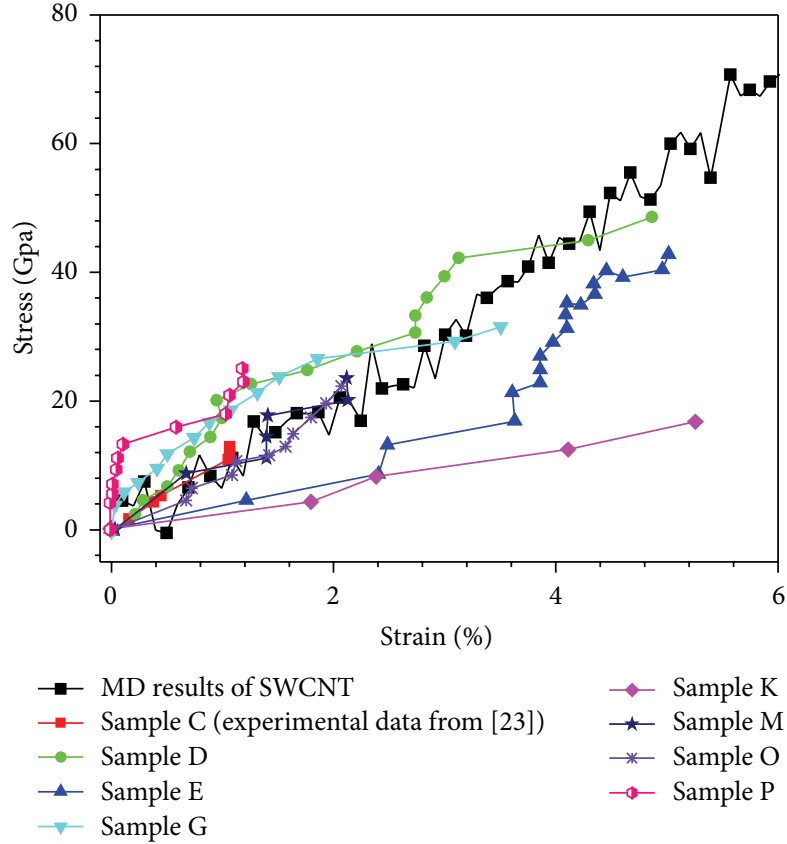

(a)

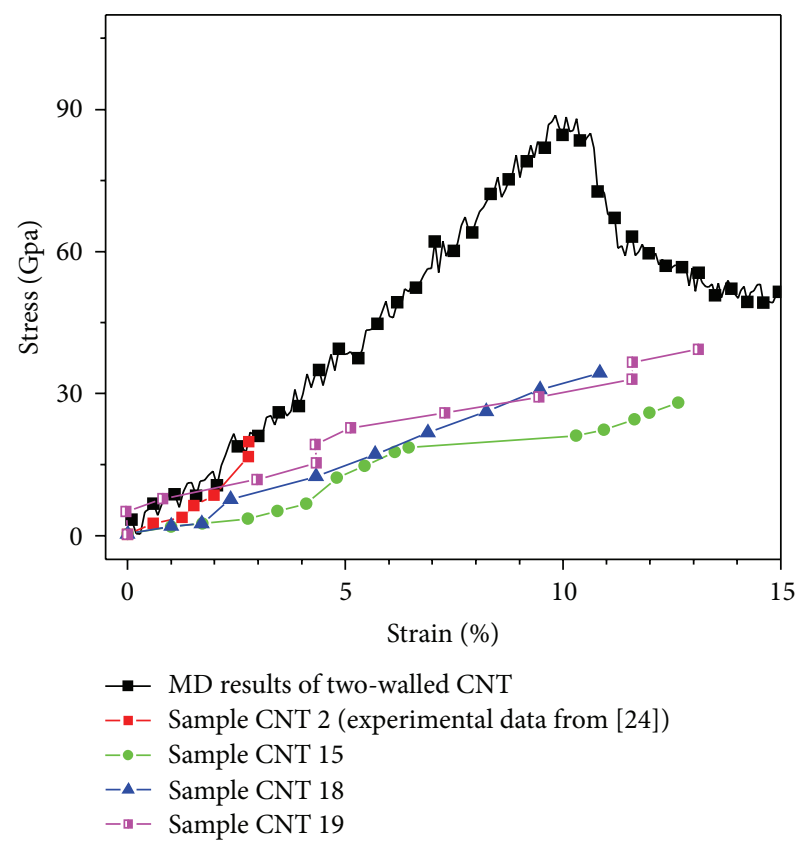

(b)

FIGURE 1: (a) The comparison of stress-strain curve of the $(6,6)$ single-walled carbon nanotube of a length $=4.92 \mathrm{~nm}$ and a diameter $=0.68 \mathrm{~nm}$ predicted by ReaxFF force field with the experimental data [23]. (b) The comparison of stress-strain curve of the $(6,6)(11,11)$ two-walled carbon nanotube of a length $=4.92 \mathrm{~nm}$ and an outer diameter $=1.5 \mathrm{~nm}$ predicted by ReaxFF force field with the experimental data [24].

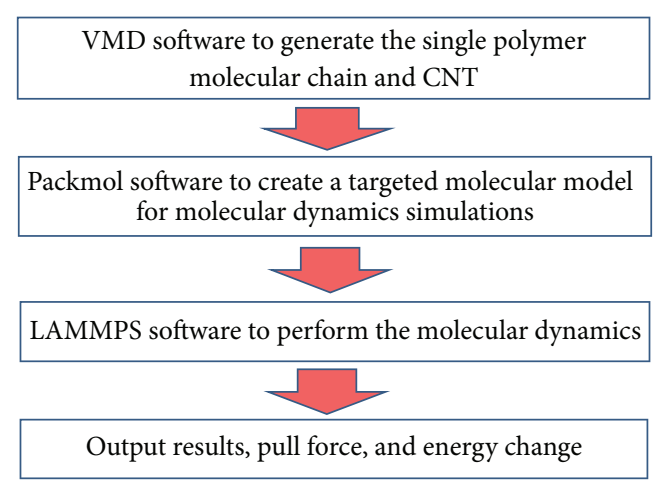

FIGURE 2: Flowchart of the simulation procedure for molecular simulation process.

nanocomposite structure with the density of $1.2 \mathrm{~g} / \mathrm{cm}^{3}$. The model of MD is shown in Figure 3.

The interfacial C-C covalent bonding is controlled by the interfacial intermolecular distance; in the present work, the $\mathrm{CNT} /$ polyethylene nanocomposite with interfacial covalent bond can be achieved as shown in Figure 3 by setting the intermolecular distance vanish.

In the MD simulations, the periodic boundary condition is applied in $x-y$ plane and the free boundary condition is assigned to $z$ direction. The time steps selected are $0.1 \mathrm{fs}$ for all the MD simulations in this present study. Before the displacement load is applied, the MD systems are equilibrated to $298 \mathrm{~K}$ and traction free by relaxing the system for 500000 steps with the use of Nose-Hoover style thermostat (NVT) and barostat (NPT) $[29,30]$ with holding the SWCNT as a rigid. And then the nanocomposite system is further putted into Nose-Hoover style thermostat (NVT) and equilibrated for 50000 steps with releasing the rigid constraints on the SWCNT.

The applying load processes in simulation are as follows: in uniaxial tension simulation, the bottom region atoms of the CNT/polyethylene nanocomposite are held fixed and the top region atoms of the nanocomposite are applied with axial displacement loads of $x=0.1 \times 10^{-4} \mathrm{~nm}$ every MD time step in the axial $(z)$ direction. In the pullout simulation, the polymer matrix is held fixed in the axial $(z)$ direction (pullout direction) and is unconstrained in $x$ and $y$ directions, applying axial displacement loads of $x=0.1 \times 10^{-4} \mathrm{~nm}$ every MD time step to all the atoms of carbon nanotube.

\section{Results and Discussion}

To test the effect of the interfacial covalent bonded interaction on the mechanical characteristics of nanocomposite, first of all, the tensile tests of the CNT/polyethylene nanocomposite of a $2.7 \mathrm{~nm} \times 2.7 \mathrm{~nm} \times 5.0 \mathrm{~nm}$ size under two different situations of with and without interfacial covalent bonded interaction were performed and the results are shown in Figure 3. In the condition of without any reinforcement Young's modulus of the polyethylene with the same density is $0.5-1.0 \mathrm{Gpa}$ and the tensile strength is about $100 \mathrm{Mpa}$; the values are too small in comparison with the results of 


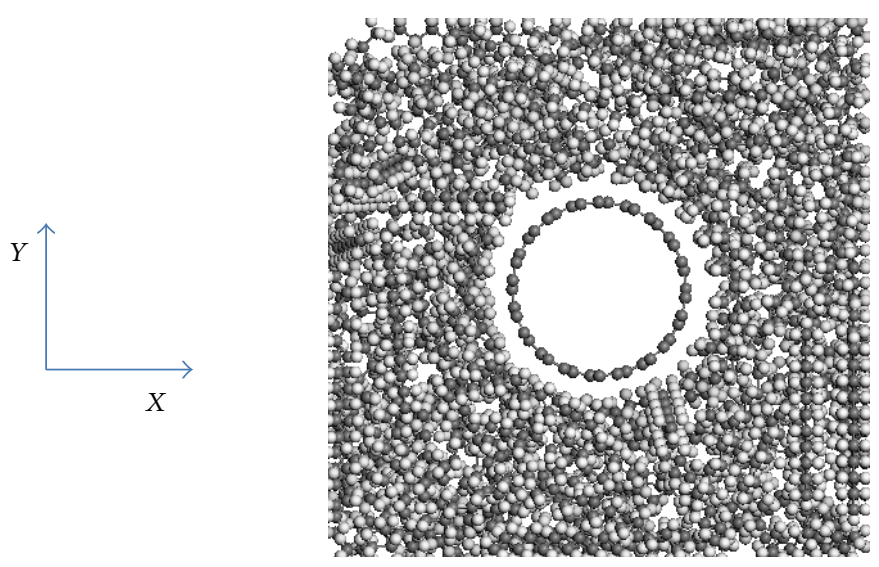

(a)

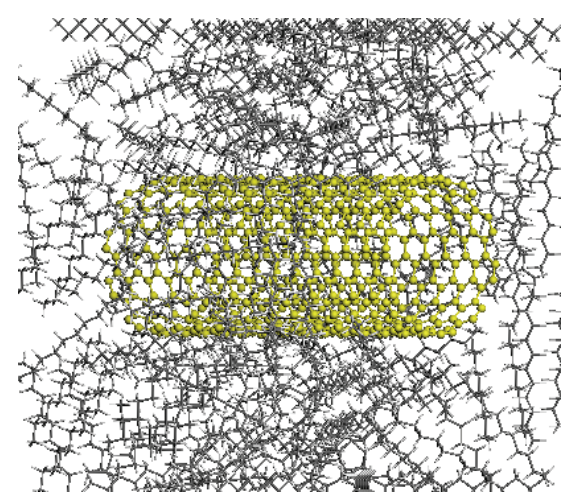

(b)

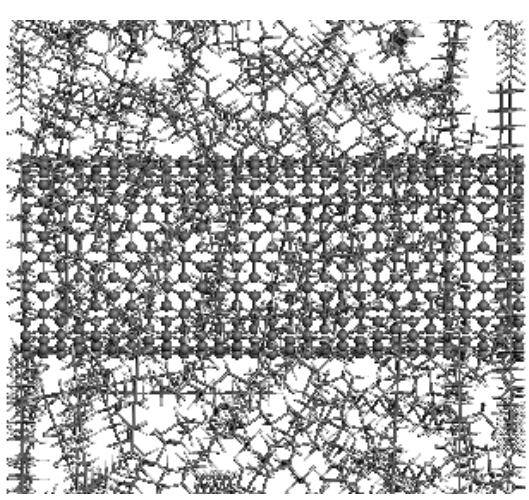

(c)

FIGURE 3: The MD model of CNT/polyethylene nanocomposite, (a) front view ( $0^{\circ}$ view), (b) lateral views $\left(45^{\circ}\right.$ view), and (c) side view $\left(90^{\circ}\right.$ view) (carbon, grey; hydrogen, white).

the nano-reinforced composites, which is the reason why there are no results of pristine polyethylene in Figure 4.

For the condition of without interfacial covalent bonded interaction, that is, only considering interfacial van der Waals interaction, estimated Young's modulus of the nanocomposite is about $13.3 \mathrm{Gpa}$ by calculating the slope ratios of the elastic stage in stress-strain curve simply. The tensile strength and the fracture strain in this condition are $1.6 \mathrm{Gpa}$ and 0.12 , respectively. Therefore, Young's modulus and the tensile strength are enhanced significantly, which are 10 times of the pristine polyethylene.

When the interfacial covalent bonded interaction is considered, that is, not only considering interfacial van der Waals interaction but also counting for the chemically bonded interaction, Young's modulus and the tensile strength of the nanocomposite are about $56.5 \mathrm{Gpa}$ and $4.6 \mathrm{Gpa}$, respectively. In addition, it is evident that for the nanocomposite there is a transition from brittle to ductile fracture with accounting for the interfacial covalent bonded interaction, which is attributed to the superior load-bearing ability of the interfacial covalent bonded interaction. And the toughness of the nanocomposite is increased dramatically. These phenomena demonstrate that the effect of interfacial covalent bonded interaction has a great influence on the mechanical characteristics of the nano-reinforced composite.
To investigate the interfacial mechanical properties of the CNTs-reinforced nanocomposite, a series of the pullout tests of CNTs from the nano-reinforced composite are carried out by using molecular dynamics method. First, a $(6,6)$ single-walled carbon nanotube is pulled out from the $\mathrm{CNT} /$ polyethylene nanocomposite of a $2.7 \mathrm{~nm} \times 2.7 \mathrm{~nm} \times$ $5.0 \mathrm{~nm}$ size with interfacial covalent bonded interaction; the results of the pullout force versus the pullout displacement and the comparison with the experimental data from the existing literature [25] are given in Figure 5. The MD simulation results of this present work agree well with the experimental data curve of the published paper, which implies that the results of this present work are credible. In the beginning stage of the pullout test, the pullout force which makes the CNT sliding in the polymer matrix is the largest part of the whole pullout process. The pullout force increases up to the maximum value promptly in a very short time (pullout distance), and with the pullout of CNTs from the polymer matrix, the area of interaction between CNTs and the polymer matrix reduces gradually, and then the pullout force decreases slowly, until it becomes zero when the CNT is pulled out from the polymer matrix completely.

The pullout force versus the pullout displacement under bonded interaction without interfacial covalent is shown in Figure 6. Obviously, the order of magnitudes of the pullout 


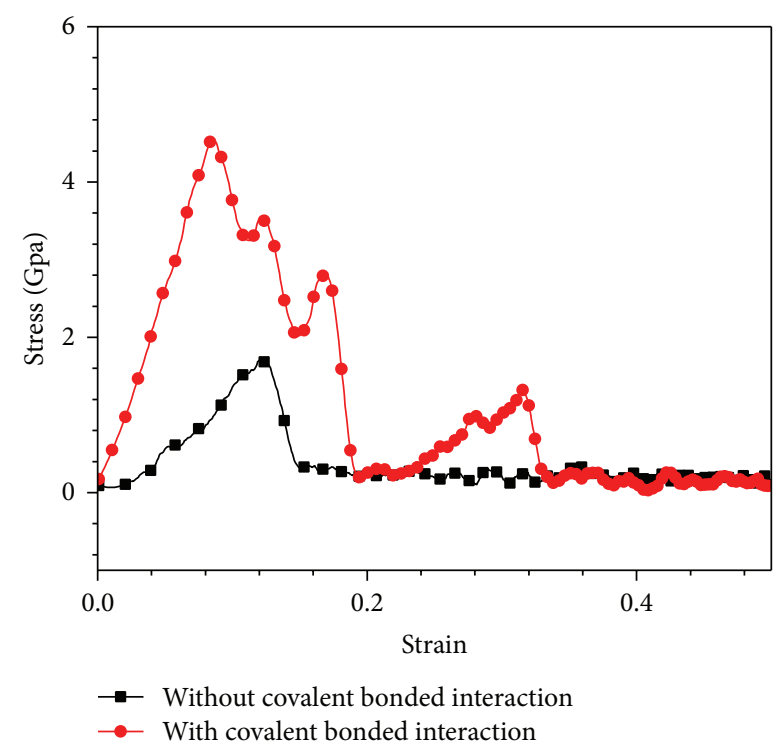

FIGURE 4: The stress-strain curves of the CNT/polyethylene nanocomposite of a $2.7 \mathrm{~nm} \times 2.7 \mathrm{~nm} \times 5.0 \mathrm{~nm}$ size predicted under two different situations of with and without interfacial covalent bonded interaction.

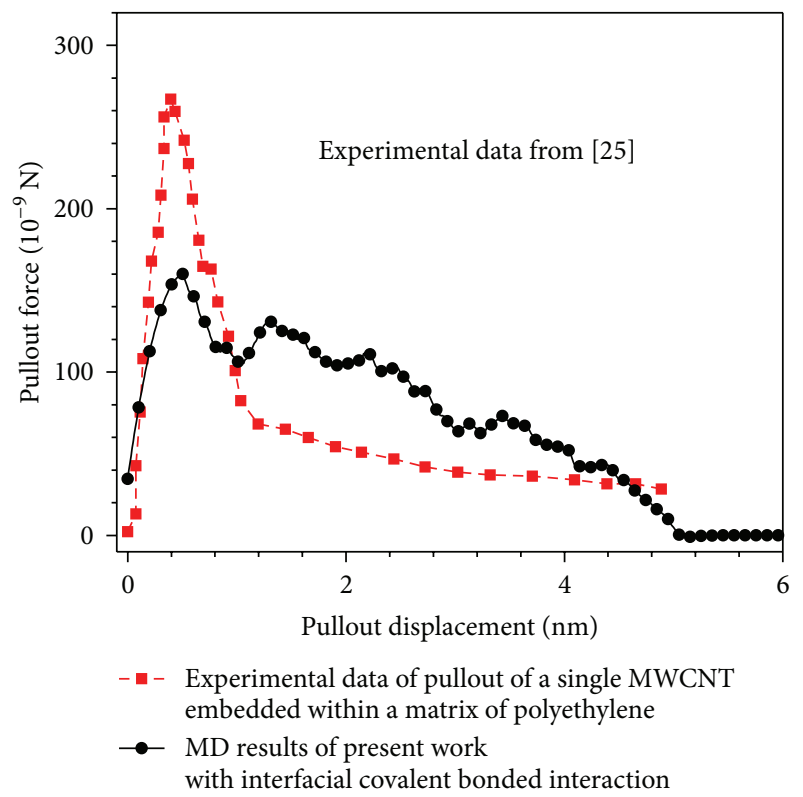

FIGURE 5: The comparison of pullout force versus pullout displacement curve of the $(6,6)$ single-walled carbon nanotube of a length $=4.92 \mathrm{~nm}$ and a diameter $=0.81 \mathrm{~nm}$ pulled out from the $\mathrm{CNT} /$ polyethylene nanocomposite of a $2.7 \mathrm{~nm} \times 2.7 \mathrm{~nm} \times 5.0 \mathrm{~nm}$ size with the experimental data [25].

force value is in the order of $10^{0} n \mathrm{~N}\left(10^{-9} \mathrm{~N}\right)$, while the order of experimental data of the existing literature mostly is in the order of $10^{2} n \mathrm{~N}\left(10^{-9} \mathrm{~N}\right)[7,8,25]$. This difference indicates that the condition of without interfacial covalent bonded interaction is very undesirable. Besides this, the effect of the CNT embedded length in the polymer has no influence on the interfacial mechanical characteristics of nanocomposite,

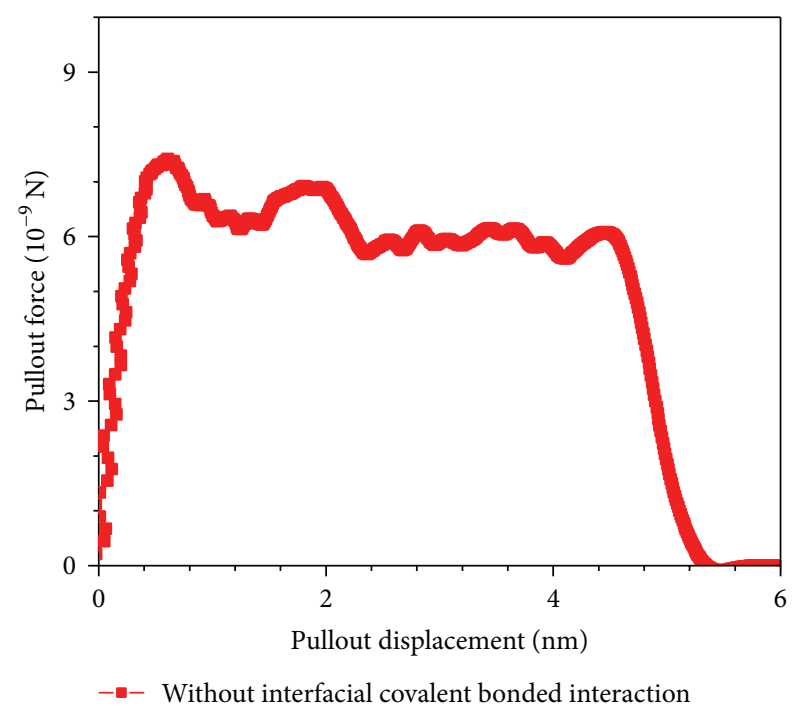

FIgURE 6: The pullout force versus pullout displacement curve of the $(6,6)$ single-walled carbon nanotube of a length $=4.92 \mathrm{~nm}$ and a diameter $=0.81 \mathrm{~nm}$ pulled out from the $\mathrm{CNT} /$ polyethylene nanocomposite of a $2.7 \mathrm{~nm} \times 2.7 \mathrm{~nm} \times 5.0 \mathrm{~nm}$ size under bonded interaction without interfacial covalent.

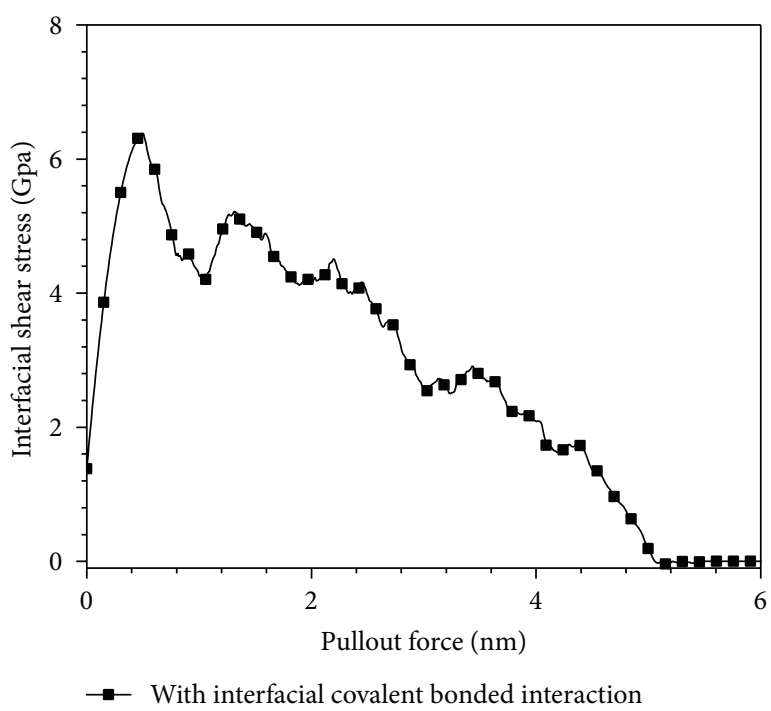

FIgURE 7: The average interfacial shear stress versus pullout displacement curve of the $(6,6)$ single-walled carbon nanotube of a length $=4.92 \mathrm{~nm}$ and a diameter $=0.81 \mathrm{~nm}$ pulled out from the CNT/polyethylene nanocomposite of a $2.7 \mathrm{~nm} \times 2.7 \mathrm{~nm} \times 5.0 \mathrm{~nm}$ size under bonded interaction with interfacial covalent.

and this phenomenon does not conform to the physical reality.

The average interfacial shear stress is estimated simply through the pullout force divided by the area of the interface (the surface area of the embedded CNT). Figure 7 shows the average interfacial shear stress for the CNT/polyethylene nanocomposite with interfacial covalent bonded interaction as a function of pullout displacement. The maximum value of interfacial shear stress appears in the beginning stage of 


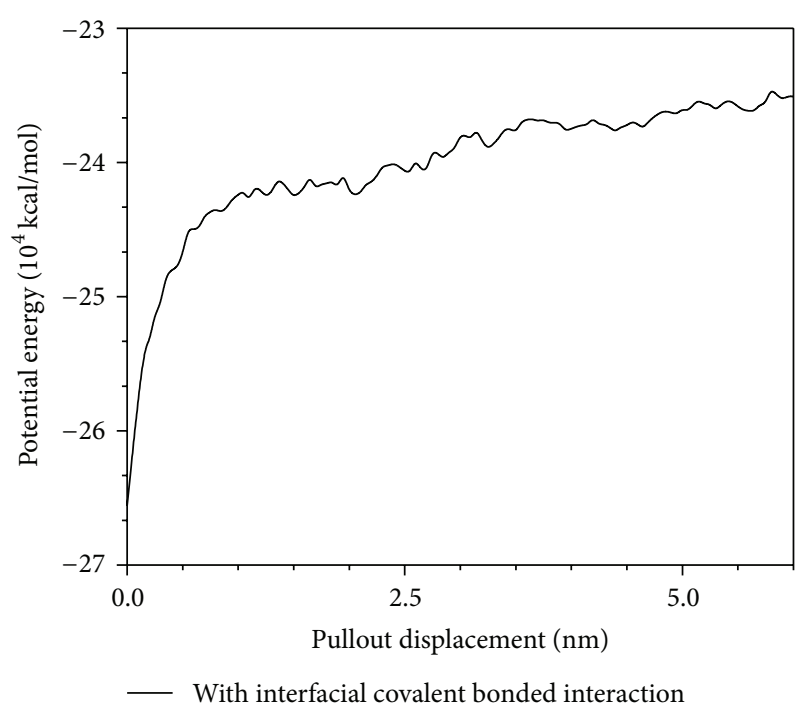

FIgURE 8: The potential energy of nanosystem versus pullout displacement curve of the $(6,6)$ single-walled carbon nanotube of a length $=4.92 \mathrm{~nm}$ and a diameter $=0.81 \mathrm{~nm}$ pulled out from the CNT/polyethylene nanocomposite of a $2.7 \mathrm{~nm} \times 2.7 \mathrm{~nm} \times 5.0 \mathrm{~nm}$ size under bonded interaction with interfacial covalent.

the pullout test, corresponding to the pullout force of this stage. With the pullout of CNTs from the polymer matrix, the interfacial shear stress reduces gradually. The estimated interfacial shear stress is in the order of a few Gpas, much greater than the modeling results of the nano-reinforced composite without interfacial covalent bonded interaction $[12,31-33]$ but is close to the results of the nanocomposites involving cross-linking using chemically bonded chains [3437]. This agreement further justifies the validity of our present MD simulation in this work.

The pullout behavior of CNTs from the polymer matrix can also be illustrated by tracking the total potential energy of the CNT/polyethylene nanocomposite system. Figure 8 shows that the total potential energy of the nanocomposite system increases as the CNT is pulled out from the polyethylene matrix, which implies the interaction between polyethylene matrix and CNT results in the variation of the total potential energy. The total potential energy ascends rapidly in the beginning stage of the pullout test and after that increases nearly linearly and slowly, which is corresponding to the pullout force in the pullout process. Due to the integrity of interface of nanocomposite in the pullout starting part, the variation of the potential energy in this stage is very large. And after this stage, because of the stable interfacial binding interaction and the steady increase of new surface area during the pullout process, the total potential energy increases nearly linearly and slowly.

The total potential energy of the nanocomposite system can be roughly divided into four terms as follows:

$$
E_{\text {potential }}=E_{\text {bond }}+E_{\text {angle }}+E_{\text {torsion }}+E_{\text {vanderWalls }} \text {. }
$$

The bond energy, the angle energy, the torsion energy, and van der Waals interaction energy (i.e., nonbond energy) of

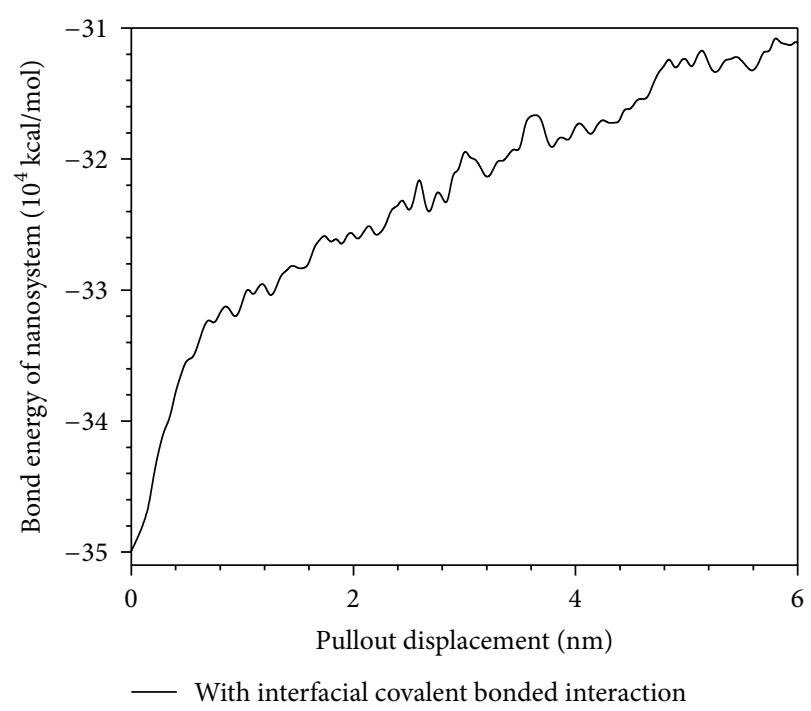

FIgURE 9: The bond energy of nanosystem versus pullout displacement curve of the $(6,6)$ single-walled carbon nanotube of a length $=4.92 \mathrm{~nm}$ and a diameter $=0.81 \mathrm{~nm}$ pulled out from the CNT/polyethylene nanocomposite of a $2.7 \mathrm{~nm} \times 2.7 \mathrm{~nm} \times 5.0 \mathrm{~nm}$ size under bonded interaction with interfacial covalent.

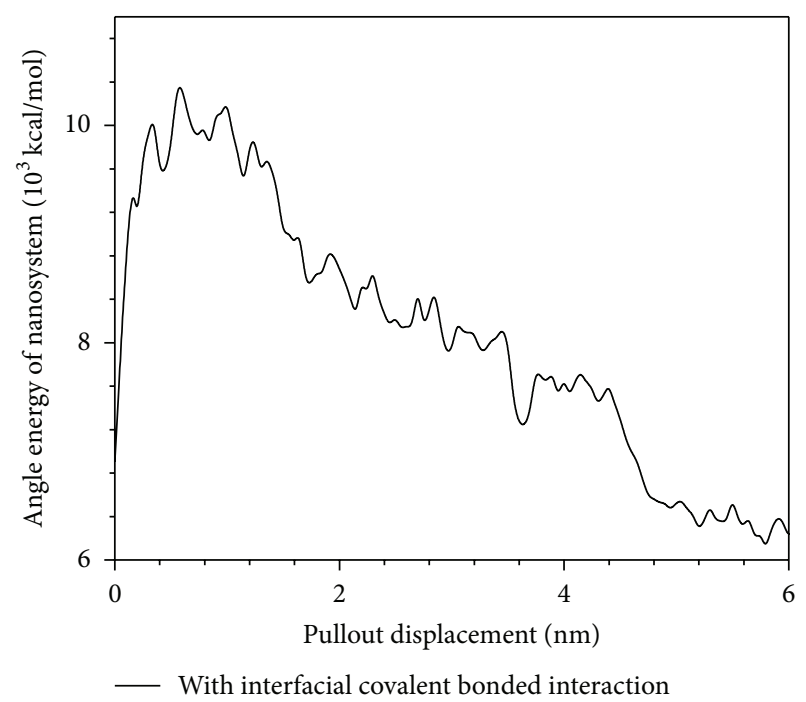

FIgURE 10: The angle energy of nanosystem versus pullout displacement curve of the $(6,6)$ single-walled carbon nanotube of a length $=4.92 \mathrm{~nm}$ and a diameter $=0.81 \mathrm{~nm}$ pulled out from the $\mathrm{CNT} /$ polyethylene nanocomposite of a $2.7 \mathrm{~nm} \times 2.7 \mathrm{~nm} \times 5.0 \mathrm{~nm}$ size under bonded interaction with interfacial covalent.

nanosystem during the pullout process are shown in Figures 9-12, respectively. Evidently in the several energy contributions of potential energy of nanosystem, the bond energy plays a significantly important role in the variation of the total potential energy, which implies that the bond interaction plays the main role in the load transfer of nanocomposites. As shown in Figure 9, the bond energy of nanosystem always increases with the pullout of CNTs from the polymer matrix; this is attributed to the bond stretch and the decrease of 


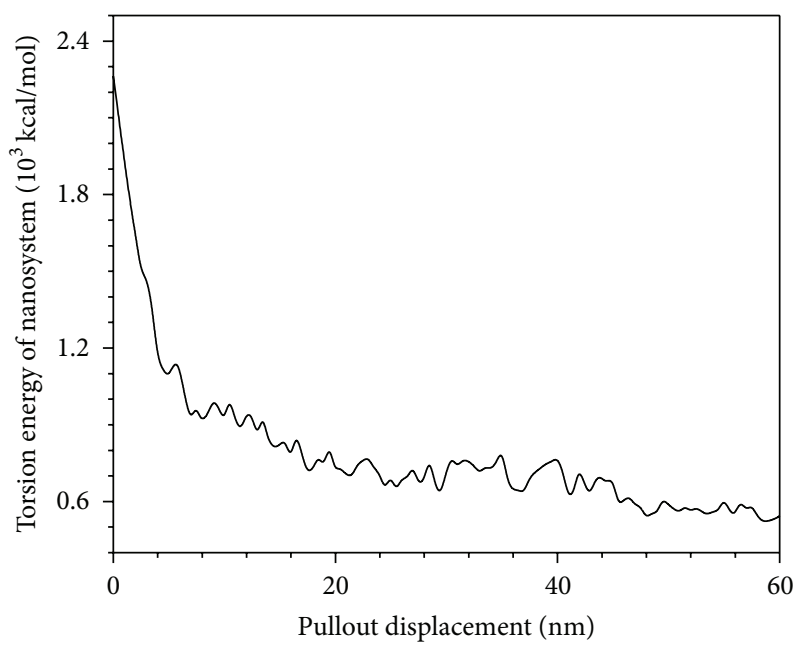

— With interfacial covalent bonded interaction

FIGURE 11: The torsion energy of nanosystem versus pullout displacement curve of the $(6,6)$ single-walled carbon nanotube of a length $=4.92 \mathrm{~nm}$ and a diameter $=0.81 \mathrm{~nm}$ pulled out from the $\mathrm{CNT} /$ polyethylene nanocomposite of a $2.7 \mathrm{~nm} \times 2.7 \mathrm{~nm} \times 5.0 \mathrm{~nm}$ size under bonded interaction with interfacial covalent.

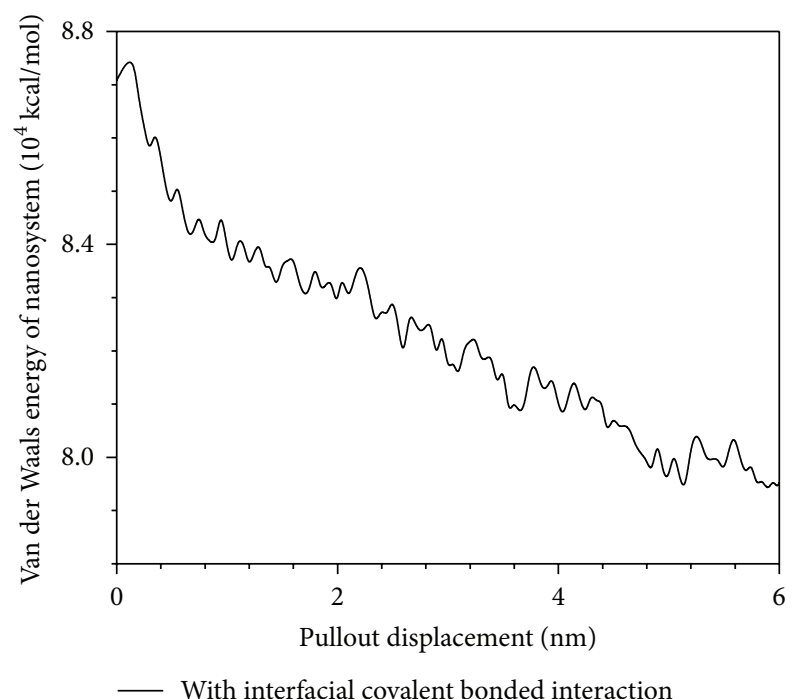

Figure 12: Van der Waals energy of nanosystem versus pullout displacement curve of the $(6,6)$ single-walled carbon nanotube of a length $=4.92 \mathrm{~nm}$ and a diameter $=0.81 \mathrm{~nm}$ pulled out from the $\mathrm{CNT} /$ polyethylene nanocomposite of a $2.7 \mathrm{~nm} \times 2.7 \mathrm{~nm} \times 5.0 \mathrm{~nm}$ size under bonded interaction with interfacial covalent.

the interface area. The angle energy, the torsion energy, and van der Waals interaction energy (i.e., nonbond energy) of nanosystem decrease during the pullout of CNTs as shown in Figures 10-12, and these energy terms will not vary after the CNTs were completely pulled out from the polymer matrix because there is no interaction between the CNTs and the polymer matrix.

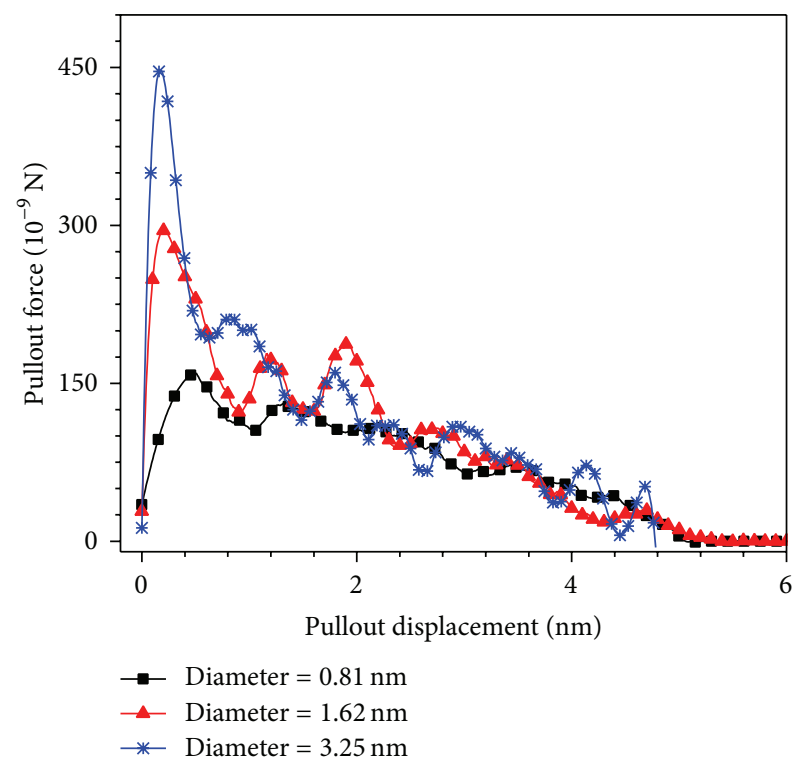

FIGURE 13: The pullout force versus pullout displacement curve of the three single-walled carbon nanotubes of a length $=4.92 \mathrm{~nm}$ and diameters $=0.81 \mathrm{~nm}, 1.62 \mathrm{~nm}$, and $3.25 \mathrm{~nm}$ pulled out from the CNT/polyethylene nanocomposites of $2.7 \mathrm{~nm} \times 2.7 \mathrm{~nm} \times 5.0 \mathrm{~nm}$ and $5.4 \mathrm{~nm} \times 5.4 \mathrm{~nm} \times 5.0 \mathrm{~nm}$ sizes under bonded interaction with interfacial covalent.

To illustrate the effect of diameter of a CNT on the interfacial mechanical properties of nanocomposite, the pullout tests for CNTs of three different diameters, $0.814 \mathrm{~nm}, 1.62 \mathrm{~nm}$, and $3.25 \mathrm{~nm}$, with a length of $4.92 \mathrm{~nm}$ embedded in the three different polymer matrices, $2.7 \mathrm{~nm} \times 2.7 \mathrm{~nm} \times 4.92 \mathrm{~nm}$, $5.4 \mathrm{~nm} \times 5.4 \mathrm{~nm} \times 4.92 \mathrm{~nm}$, and $10.8 \mathrm{~nm} \times 10.8 \mathrm{~nm} \times 4.92 \mathrm{~nm}$, with the same density are simulated; the pull force versus the pullout displacement is shown in Figure 13. In the beginning stage of the pullout tests, the diameter of CNTs has a great influence on the pullout force. But in the following pullout progress, there seems to be no distinguished difference in the results of three different models. These results indicate that the diameter of CNTs in the nano-reinforced composite only affects the starting pullout force of CNTs and has a slight influence on the pullout force of the following pullout stage.

To demonstrate the effect of length of a CNT on the interfacial mechanical properties of nanocomposite, the pullout tests for CNTs of three different lengths, $4.92 \mathrm{~nm}, 9.85 \mathrm{~nm}$, and $12.31 \mathrm{~nm}$, with a diameter of $0.81 \mathrm{~nm}$ embedded in the three different polymer matrices, $2.7 \mathrm{~nm} \times 2.7 \mathrm{~nm} \times 5.0 \mathrm{~nm}$, $2.7 \mathrm{~nm} \times 2.7 \mathrm{~nm} \times 10 \mathrm{~nm}$, and $2.7 \mathrm{~nm} \times 2.7 \mathrm{~nm} \times 15 \mathrm{~nm}$, with the same density are simulated; the pull force versus the pullout displacement is shown in Figure 14. In the whole process of the pullout tests, the length of CNTs has a great influence on the pullout force. With the pullout of CNTs from the polymer matrix, the difference reduces gradually. These results show that the length of CNTs in the nano-reinforced composite has a great influence on the pullout force of the whole pullout stage. 


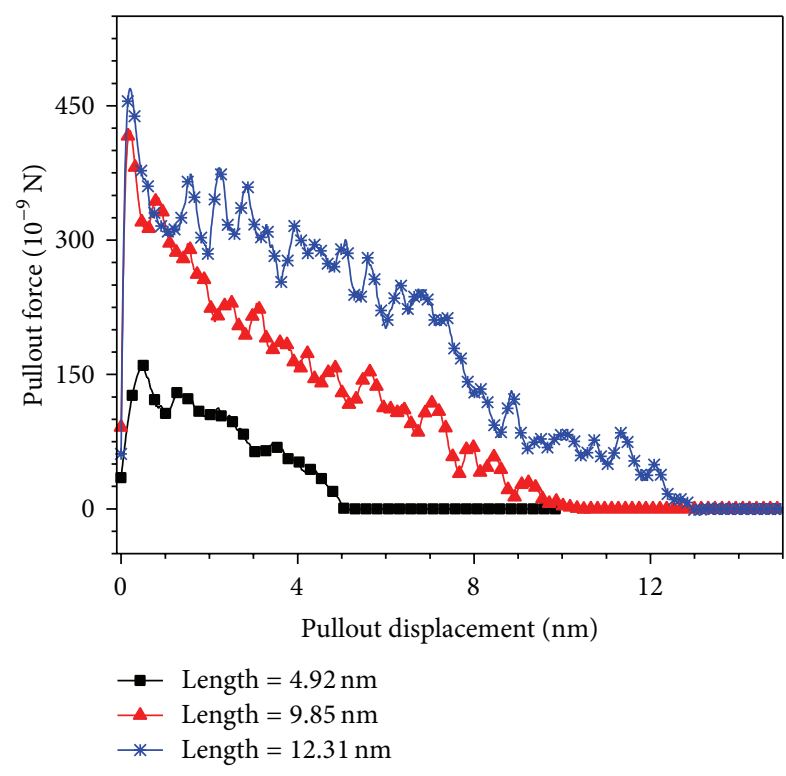

FIGURE 14: The pullout force versus pullout displacement curve of the three single-walled carbon nanotube of a diameter $=0.81 \mathrm{~nm}$ and lengths $=4.92 \mathrm{~nm}, 9.85 \mathrm{~nm}$, and $12.31 \mathrm{~nm}$ pulled out from the CNT/polyethylene nanocomposites of $2.7 \mathrm{~nm} \times 2.7 \mathrm{~nm} \times 5.0 \mathrm{~nm}$, $2.7 \mathrm{~nm} \times 2.7 \mathrm{~nm} \times 10.0 \mathrm{~nm}$, and $2.7 \mathrm{~nm} \times 2.7 \mathrm{~nm} \times 15.0 \mathrm{~nm}$ sizes under bonded interaction with interfacial covalent.

\section{Concluding Remarks}

With accounting for interfacial covalent bonded interaction, the mechanical properties of carbon nanotube- (CNT-) reinforced polyethylenes (PE) are investigated using molecular dynamics simulations. The results show that the interfacial covalent bonded interaction between CNTs and the polymer matrix is indispensable. The bond interaction plays the main role in the load transfer of nanocomposites. In addition, the effects of carbon nanotube embedded length and diameter have great influences on the interfacial mechanical properties of nano-reinforced composite.

\section{Conflict of Interests}

The authors declare that there is no conflict of interests regarding the publication of this paper.

\section{Acknowledgments}

This work is supported by the Fundamental Research Funds for the Central Universities (2015QN140) and National Science Foundation of China (11172230, 11372240).

\section{References}

[1] M. M. J. Treacy, T. W. Ebbesen, and J. M. Gibson, "Exceptionally high Young's modulus observed for individual carbon nanotubes," Nature, vol. 381, no. 6584, pp. 678-680, 1996.

[2] B. I. Yakobson, C. J. Brabec, and J. Bernholc, "Nanomechanics of carbon tubes: instabilities beyond linear response," Physical Review Letters, vol. 76, no. 14, pp. 2511-2514, 1996.
[3] J. W. Che, T. Çagin, and W. A. Goddard III, “Thermal conductivity of carbon nanotubes," Nanotechnology, vol. 11, no. 2, pp. 65-69, 2000.

[4] S. Berber, Y.-K. Kwon, and D. Tománek, "Unusually high thermal conductivity of carbon nanotubes," Physical Review Letters, vol. 84, no. 20, pp. 4613-4616, 2000.

[5] T. W. Ebbesen, H. J. Lezec, H. Hiura, J. W. Bennett, H. F. Ghaemi, and T. Thio, "Electrical conductivity of individual carbon nanotubes," Nature, vol. 382, no. 6586, pp. 54-56, 1996.

[6] J. N. Coleman, U. Khan, W. J. Blau, and Y. K. Gun'ko, "Small but strong: a review of the mechanical properties of carbon nanotube-polymer composites," Carbon, vol. 44, no. 9, pp. 16241652, 2006.

[7] C. A. Cooper, S. R. Cohen, A. H. Barber, and H. D. Wagner, "Detachment of nanotubes from a polymer matrix," Applied Physics Letters, vol. 81, no. 20, pp. 3873-3875, 2002.

[8] A. A. Koval'chuk, V. G. Shevchenko, A. N. Shchegolikhin, P. M. Nedorezova, A. N. Klyamkina, and A. M. Aladyshev, "Effect of carbon nanotube functionalization on the structural and mechanical properties of polypropylene/MWCNT composites," Macromolecules, vol. 41, no. 20, pp. 7536-7542, 2008.

[9] S. J. V. Frankland, A. Caglar, D. W. Brenner, and M. Griebel, "Molecular simulation of the influence of chemical cross-links on the shear strength of carbon nanotube-polymer interfaces," The Journal of Physical Chemistry B, vol. 106, no. 12, pp. 30463048, 2002.

[10] N. Lachman and H. D. Wagner, "Correlation between interfacial molecular structure and mechanics in CNT/epoxy nanocomposites," Composites Part A: Applied Science and Manufacturing, vol. 41, no. 9, pp. 1093-1098, 2010.

[11] F. Buffa, G. A. Abraham, B. P. Grady, and D. Resasco, "Effect of nanotube functionalization on the properties of single-walled carbon nanotube/polyurethane composites," Journal of Polymer Science, Part B: Polymer Physics, vol. 45, no. 4, pp. 490-501, 2007.

[12] Q. Zheng, D. Xia, Q. Xue, K. Yan, X. Gao, and Q. Li, "Computational analysis of effect of modification on the interfacial characteristics of a carbon nanotube-polyethylene composite system," Applied Surface Science, vol. 255, no. 6, pp. 3534-3543, 2009.

[13] Q. Zheng, Q. Xue, K. Yan, X. Gao, Q. Li, and L. Hao, "Effect of chemisorption on the interfacial bonding characteristics of carbon nanotube-polymer composites," Polymer, vol. 49, no. 3, pp. 800-808, 2008.

[14] A. H. Barber, S. R. Cohen, and H. D. Wagner, "Measurement of carbon nanotube-polymer interfacial strength," Applied Physics Letters, vol. 82, no. 23, pp. 4140-4142, 2003.

[15] B. Vigolo, V. Mamane, F. Valsaque et al., "Evidence of sidewall covalent functionalization of single-walled carbon nanotubes and its advantages for composite processing," Carbon, vol. 47, no. 2, pp. 411-419, 2009.

[16] Z. Huang, L. Xi, Q. Subhani, W. Yan, W. Guo, and Y. Zhu, "Covalent functionalization of multi-walled carbon nanotubes with quaternary ammonium groups and its application in ion chromatography," Carbon, vol. 62, pp. 127-134, 2013.

[17] L. S. Fifield and J. W. Grate, "Hydrogen-bond acidic functionalized carbon nanotubes (CNTs) with covalently-bound hexafluoroisopropanol groups," Carbon, vol. 48, no. 7, pp. 2085-2088, 2010.

[18] M. Ghosh, S. Maiti, S. Dutta, D. Das, and P. K. Das, "Covalently functionalized single-walled carbon nanotubes at reverse micellar interface: a strategy to improve lipase activity," Langmuir, vol. 28, no. 3, pp. 1715-1724, 2012. 
[19] H. Hu, B. Yu, Q. Ye, Y. Gu, and F. Zhou, "Modification of carbon nanotubes with a nanothin polydopamine layer and polydimethylamino-ethyl methacrylate brushes," Carbon, vol. 48, no. 8 , pp. 2347-2353, 2010.

[20] A. Peyvandi, P. Soroushian, N. Abdol, and A. M. Balachandra, "Surface-modified graphite nanomaterials for improved reinforcement efficiency in cementitious paste," Carbon, vol. 63, pp. 175-186, 2013.

[21] Q. L. Xiong and X. G. Tian, "Atomistic simulations of interfacial mechanical characteristics of carbon nanotube/silicon nanocomposites," Molecular Simulation, 2014.

[22] A. C. T. van Duin, S. Dasgupta, F. Lorant, and W. A. Goddard III, "ReaxFF: a reactive force field for hydrocarbons," The Journal of Physical Chemistry A, vol. 105, no. 41, pp. 9396-9409, 2001.

[23] M.-F. Yu, B. S. Files, S. Arepalli, and R. S. Ruoff, “Tensile loading of ropes of single wall carbon nanotubes and their mechanical properties," Physical Review Letters, vol. 84, no. 24, pp. 5552$5555,2000$.

[24] M.-F. Yu, O. Lourie, M. J. Dyer, K. Moloni, T. F. Kelly, and R. S. Ruoff, "Strength and breaking mechanism of multiwalled carbon nanotubes under tensile load," Science, vol. 287, no. 5453, pp. 637-640, 2000.

[25] A. H. Barber, S. R. Cohen, S. Kenig, and H. D. Wagner, "Interfacial fracture energy measurements for multi-walled carbon nanotubes pulled from a polymer matrix," Composites Science and Technology, vol. 64, no. 15, pp. 2283-2289, 2004.

[26] S. Nosé, "A unified formulation of the constant temperature molecular dynamics methods," The Journal of Chemical Physics, vol. 81, no. 1, pp. 511-519, 1984.

[27] S. Plimpton, "Fast parallel algorithms for short-range molecular dynamics," Journal of Computational Physics, vol. 117, no. 1, pp. 1-19, 1995.

[28] W. Humphrey, A. Dalke, and K. Schulten, "VMD: visual molecular dynamics," Journal of Molecular Graphics, vol. 14, no. 1, pp. 33-38, 1996.

[29] http://www.ime.unicamp.br/ martinez/packmol/.

[30] W. G. Hoover, "Canonical dynamics: equilibrium phase-space distributions," Physical Review A, vol. 31, no. 3, pp. 1695-1697, 1985.

[31] S. J. V. Frankland, A. Caglar, D. W. Brenner, and M. Griebel, "Molecular simulation of the influence of chemical cross-links on the shear strength of carbon nanotube-polymer interfaces," Journal of Physical Chemistry B, vol. 106, no. 12, pp. 3046-3048, 2002.

[32] X. Xu, M. M. Thwe, C. Shearwood, and K. Liao, "Mechanical properties and interfacial characteristics of carbon-nanotubereinforced epoxy thin films," Applied Physics Letters, vol. 81, no. 15, pp. 2833-2836, 2002.

[33] J. M. Wernik, B. J. Cornwell-Mott, and S. A. Meguid, "Determination of the interfacial properties of carbon nanotube reinforced polymer composites using atomistic-based continuum model," International Journal of Solids and Structures, vol. 49, no. 13, pp. 1852-1863, 2012.

[34] F. Pavia and W. A. Curtin, "Interfacial sliding in carbon nanotube/diamond matrix composites," Acta Materialia, vol. 59, no. 17, pp. 6700-6709, 2011.

[35] S. C. Chowdhury and T. Okabe, "Computer simulation of carbon nanotube pull-out from polymer by the molecular dynamics method," Composites A: Applied Science and Manufacturing, vol. 38, no. 3, pp. 747-754, 2007.
[36] S. Namilae and N. Chandra, "Multiscale model to study the effect of interfaces in carbon nanotube-based composites," Journal of Engineering Materials and Technology, vol. 127, no. 2, pp. 222-232, 2005.

[37] S. Namilae, N. Chandra, and C. Shet, "Mechanical behavior of functionalized nanotubes," Chemical Physics Letters, vol. 387, no. 4-6, pp. 247-252, 2004. 

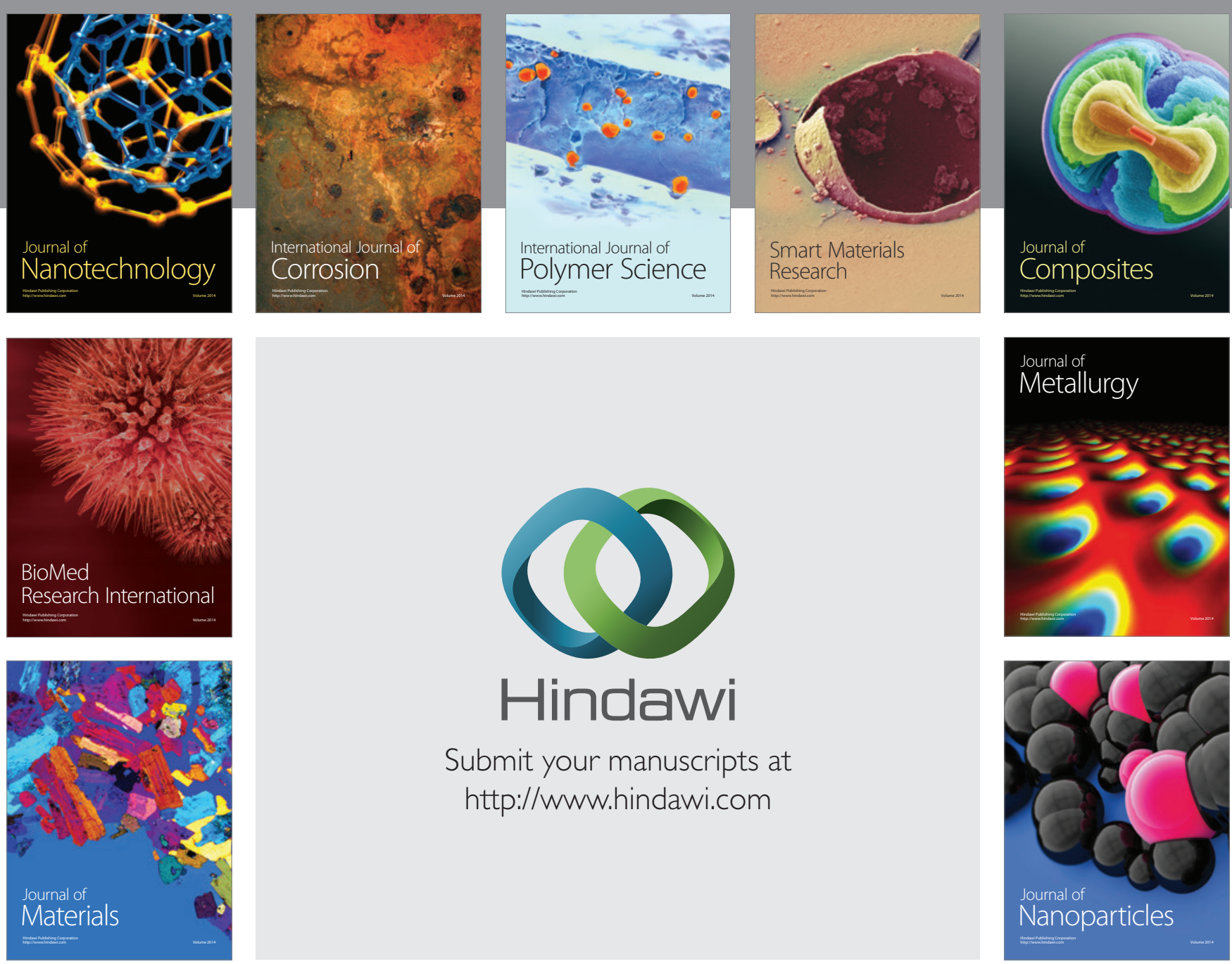

Submit your manuscripts at http://www.hindawi.com
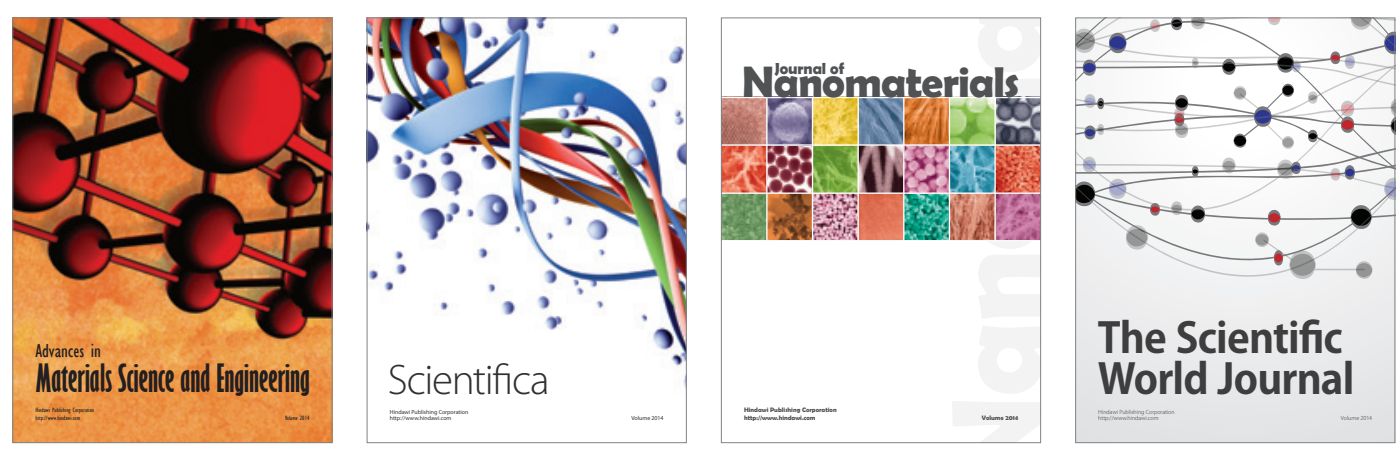

\section{The Scientific World Journal}
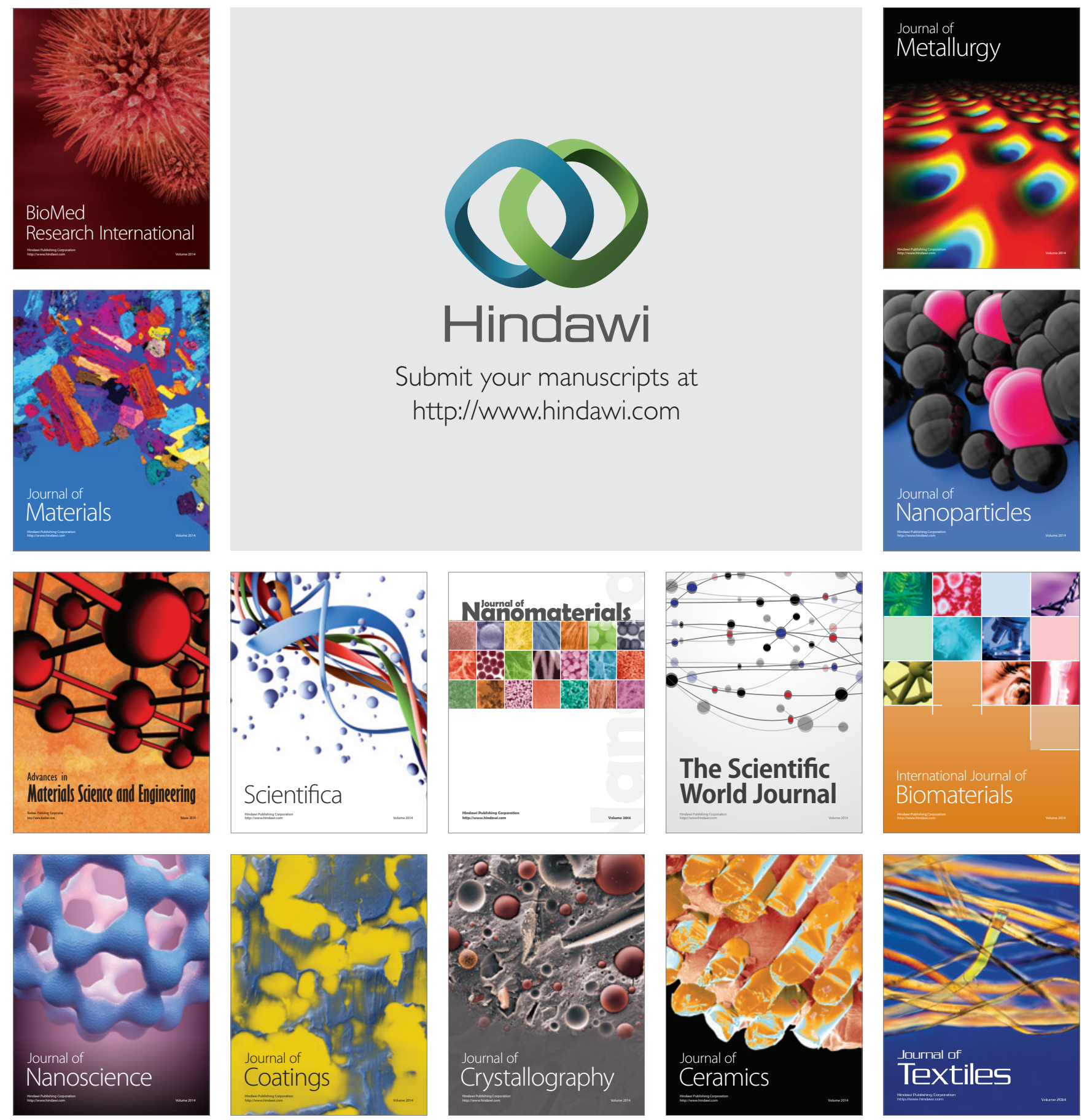